\title{
Chronic Fatigue Syndrome (CFS): An Imprecisely Defined Infectious Disease Caused by Stealth Adapted Viruses
}

\author{
W John Martin* \\ Institute of Progressive Medicine, South Pasadena, USA
}

*Corresponding author: W John Martin, Institute of Progressive Medicine, 1634 Spruce Street, South Pasadena, CA 91030, USA

\section{Introduction}

Much of the research on the chronic fatigue syndrome (CFS) is misguided for two major reasons. First, it is falsely assumed that CFS is a distinct, definable illness, which can be reliably differentiated from other neurological and psychiatric illnesses. Second, in spite of compelling evidence to the contrary, CFS is not generally regarded as an infectious illness. This review addresses these two issues and is followed by a brief discussion on stealth adapted viruses and the alternative cellular energy (ACE) pathway.

\section{Diagnosis}

It is clearly inappropriate that the diagnosis of CFS is being applied to severely ill, bedridden patients, as well as to outspoken, internet savvy individuals who frequently attend conferences and other public events. The core symptom for the majority of those diagnosed with CFS is a feeling of not having sufficient physical and/or mental capacity to undertake ordinary tasks, with no clear explanation as to why this limitation exists. The frustrating feeling of persisting fatigue will typically become exacerbated, with a delayed onset after even modest physical and/or mental exertion and can remain so for several days, without being relieved by sleep. Beyond the core symptom of unexplained fatigue, there are a plethora of additional clinical manifestations. To a varying extent, different CFS patients experience mental confusion with a lack of clarity in their thoughts (brain fog); inability to stay focused on a topic (attention deficit); poor memory, even forgetting the names of common everyday items; and emotional lability, ex- pressed as periods of sadness (lack of joy); and occasionally as anxiety, anger and/or depression. CFS patients can often experience hypersensitivity to sensory inputs, leading to pain, paresthesia, photophobia, tinnitus and chemical sensitivity. The autonomic nervous system may show signs of dysregulation such as postural hypotension with tachycardia; other disorders affecting blood circulation; irritable bowel; etc. The severity of these various symptoms differs widely among patients diagnosed with CFS and can also vary markedly over time in the same individual. Unless further defined, the CFS diagnosis is largely uninformative with regards to a particular patient's illness. Furthermore, similar sets of symptoms can be present in patients with traumatic brain injuries and in patients in whom additional symptoms or laboratory test results can lead to a better defined neurological, psychiatric, or autoimmune diagnosis. Based on more questionable criteria, some patients who would otherwise be diagnosed as mild to moderate CFS are diagnosed as having depression, personality disorder, burnout, post-traumatic stress, fibromyalgia, chronic Lyme disease, Gulf War syndrome, chronic inflammatory response syndrome (CIRS), chemical sensitivity, electromagnetic sensitivity, irritable bowel syndrome, etc. According to some definitions, CFS is excluded if the patient has psychiatric symptoms, yet other definitions allow for co-morbid psychiatric diagnoses [1-6].

Several laboratory tests will commonly yield results in CFS patients, which are different from those in most healthy controls [7-26]. The items being tested are only loosely directed to the element of chronic

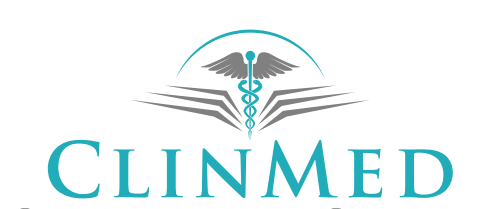

INTERNATIONAL LIBRARY 
fatigue. Moreover, similar abnormal results can occur in association with many other chronic illnesses in which fatigue is not a predominant feature. Emotional stress and prolonged periods of physical inactivity can potentially cause disruptions in the biochemical pathways measured in some of these assays. It has also been argued that knowing that one has an abnormal test result will simply add to further pessimism about being ill. Certain individuals seem to do better by consciously disregarding the illness concept and are more accepting of their physical and mental limitations. The diagnosis of CFS for these patients is considered counterproductive.

\section{Infection}

Multiple lines of evidence indicate an infectious cause of CFS. The first is the occurrence of major outbreaks of CFS-related illnesses. Seventy-three outbreaks occurring between 1934 and 1990 are listed in the book The Clinical and Scientific Basis of Myalgic Encephalomyelitis - Chronic Fatigue Syndrome, edited by Dr. Byron Hyde [27]. Prominent among these outbreaks was an illness beginning in 1985 and first publicly reported in 1986, which occurred in the Lake Tahoe/Incline Village region of Nevada [28]. Two of the many unpublicized subsequent outbreaks in the US are worth noting. Dr. Donovan Anderson manages a general medical practice in Mohave Valley, Arizona, which is nearby to Needles, California. In the spring of 1996, he became aware of an acute illness which progressively affected well over a hundred of the local residents. Individuals presented with acute gastrointestinal symptoms, developing approximately a week after contact with someone with similar symptoms. Vomiting, diarrhea and abdominal pain would resolve within 1-2 weeks. Rather than the individuals regaining their prior health, most progressed to a chronic illness. This illness was characterized by severe fatigue, cognitive impairments and mood disorders [29]. The disease affecting most of these individuals was confirmed as CFS by a visiting specialist in this condition. Several patients had very severe illnesses, including dementia. A veterinarian exhibited symptoms of acute depression prior to suicide. An 8-yearold child of a symptomatic mother exhibited attention deficit hyperactivity disorder (ADHD) in second grade. He mentioned to his mother that he would see double when tired or stressed. An MRI of his brain showed fairly extensive white matter disease suggestive of a leukodystrophy, with frontal lobe predominance. A brain biopsy of the frontal lobe showed no inflammation. Rather it was pathologically described as a spongy (vacuolar) myelinopathy [30]. Based on a positive stealth virus culture (to be discussed in a later section of this article), the child was prescribed a course of ganciclovir, which provided some temporary clinical improvements. Nevertheless, he continued to deteriorate, became physically incapacitated and died approximately one-year later. Blood samples from over 50 additional patients involved in this outbreak were also cultured for evidence of infection with stealth adapted viruses. All of the tested samples gave strongly positive results. Most of the patients have remained chronically ill for over 20 years with many now being diagnosed as having fibromyalgia in addition to CFS.

A hairdresser in Joelton, TN, realized she, her two staff members, and a number of her clients were experiencing episodes of pain that mainly affected areas of their skin, along with a feeling of general malaise and noticeably impaired thought processing. She communicated her concerns to the local health authority and to the Centers for Disease Control and Prevention $(C D C)$. The representative from the $C D C$ asked if she knew of additional patients. The hairdresser took the initiative of describing her illness in the local Shopper magazine. Well over 100 people from the local community responded that they were experiencing similar symptoms. As soon as the issue of a potential infectious process arose, the local health authority, on the advice from CDC, refused to engage in any further contact. This patient and several of her close associates tested positive in stealth adapted virus cultures.

\section{IIIness among Family Members}

The second compelling argument for an infectious cause of CFS is the occurrence of diverse illnesses among family members of presumptive infectious origin. Various families can trace the sequential onset of illness among the various family members. A woman in Florida with CFS was convinced that her husband had developed the same illness. For economic reasons and despite the added hardships, he was in denial of his illness in order to stay employed. The woman's mother developed Parkinson's disease and her son was diagnosed with schizophrenia. Yet they could all relate to the overlapping fatigue and brain fog, which were affecting them all.

A banker had to retire due to early onset of dementia. His wife tried to provide support, but she too became incapacitated with hyperesthesia and paranoia. Their daughter agreed to accommodate her parents in her home. Soon, thereafter, she developed CFS and her husband developed amyotrophic lateral sclerosis (ALS). Each of their four children became sick, one with thrombocytopenia and all with learning and behavioral disorders. Extended family members also became symptomatic.

A gentleman returned from an overseas business trip and developed a fatiguing illness. Although there was no intercourse during his travel, he was concerned with the possibility that he might have acquired an HIV infection. The laboratory tests for HIV came back nega- 
tive. His elderly father became ill a week after visiting his son. He was aware of published work showing that certain stealth adapted viruses had originated from African green monkey simian cytomegalovirus (SCMV). He took the initiative of pretending to be a veterinarian caring for sick primates. He did so to order testing for monkey cytomegaloviruses from an animal diagnostic testing laboratory in Los Angeles. To support the pretense, he had his and his father's blood labeled using the names of the two dogs belonging to his neighbor. The results were that both of the blood samples were positive by the polymerase chain reaction (PCR) for SCMV. The gentleman then notified me of the testing and he and I reported the result to the CDC. They arranged for a blood sample to be sent to a private clinical laboratory in Atlanta, where the sample was said to be virus negative.

\section{Illnesses in Animals}

The third and most convincing line of evidence began as an animal study conducted by Dr. Tom Glass at the University of Oklahoma. He recorded the high incidence of illnesses in the dogs and cats of CFS patients [31,32]. The pet owners could sometimes relate their symptoms with those developing in a newly acquired animal pet. Interviews with CFS patients will not uncommonly describe situations such as a previously healthy cat becoming unsteady when walking on the back of a couch, or seemingly not recalling where their food is usually provided. One patient's cat was even spooked upon seeing a garden hose. More overt neurological signs would subsequently appear in many of the affected pets leading to euthanasia. In conjunction with Dr. Glass, a stealth adapted virus cultured from a CFS patient was inoculated into six healthy cats. Within a few days, all of the cats became acutely ill [33]. They became reclusive, avoided light, would commonly rub their head against the cage, leading to a loss of fur from the rubbed areas. They were very sensitive to being held. On necropsy, there were vacuolated, degenerative cells throughout the brain. Yet there was no inflammation in the brain or elsewhere in the body. The acute illness peaked between 2 and 4 weeks, after which there was clinical recovery. There were stillborn births to a pregnant cat [31-33].

\section{Stealth Adapted Viruses}

Stealth adapted viruses differ from the viruses from which they are derived in not evoking inflammation [34-40]. This is because of the deletion or mutation in the genes coding for the relatively few virus components normally targeted by the cellular immune system. The prototype stealth adapted virus is a derivative of SCMV $[41,42]$. It has a genetically unstable, fragmented genome [43]. The various fragments are unevenly distributed in the remaining genome. Moreover, several cellular sequences have been incorporated into the replicating virus genome
[44]. So too have some bacterial sequences [45].The cellular-derived sequences are from non-coding regions of the cellular genome. The bacteria-derived sequences, in this well characterized SCMV-derived stealth adapted virus, are from mycoplasma and from one or more atypical alpha-proteobacteria [46]. The term renegade is being used to describe the incorporated cellular and bacterial sequences [46]. Renegade sequences have been identified in other stealth adapted viruses. They become part of the virus replication process and are transmitted between cells, individuals, and even species, as components of reformed virus genomes. The renegade sequences can potentially replace all of the sequences of the initiating virus. This can have two major consequences. First, it can explain why CFS has been mistaken as a bacterial infection, e.g. due to mycoplasma or brucella [47]. Second, it means that relying upon the presence of established immunological and/or molecular markers for known human and animal viruses can lead to false negative results [48]. The most reliable way of detecting stealth adapted viruses is by virus culture. CFS patients almost invariably test positive in specialized virus cultures [29,39-42]. The cultures also provide a useful test system to assess anti-virus therapies.

\section{The Alternative Cellular Energy (ACE) Pathway}

While the particular cell-derived and bacteria-derived sequences in the different stealth adapted viruses may contribute directly to the virus-induced cell damage, the more basic issue in virus infections is that the virus is competing for cellular resources. Virus replication requires cellular energy. So too do the cellular anti-virus defenses and cellular repair mechanisms. It was wrongly assumed that cellular energy is solely provided from the metabolism of food [40]. The body can attract additional energy from an environmental force provisionally termed KELEA (Kinetic Energy Limiting Electrostatic Attraction). The fundamental role of this force is presumably to prevent the fusion and annihilation of electrostatically attracted opposite electrical charges. The fluctuating electrical activity of the brain and possibly muscles can attract KELEA from the environment as a non-food source of cellular energy [49]. This energy is expressed as an added kinetic quality of the body's fluids in what is referred to as the alternative cellular energy (ACE) pathway. KELEA can also be transferred to water for consumption or external application in humans. Indeed, small pouches of KELEA activated water can simply be worn close to the body as a means of enhancing the ACE pathway [50]. Combining methods for improving the KELEA receiving function of the brain with the use of KELEA activated water provides a rational approach to overcoming stealth adapted virus infections. It is the preferred approach for treat- 
ing patients diagnosed as having CFS and for patients with the many other possible clinical manifestations of being infected with a stealth adapted virus.

\section{Acknowledgement}

The work is supported by MI Hope Inc., a non-profit public charity.

\section{References}

1. Morris G, Maes M (2013) Case definitions and diagnostic criteria for myalgic encephalomyelitis and chronic fatigue syndrome: From clinical-consensus to evidence-based case definitions. Neuro Endocrinol Lett 34: 185-199.

2. Brurberg KG, Fonhus MS, Larun L, Flottorp S, Malterud K (2014) Case definitions for chronic fatigue syndrome/myalgic encephalomyelitis (CFS/ME): A systematic review. BMJ Open 4: e003973.

3. Bested AC, Marshall LM (2015) Review of myalgic encephalomyelitis/chronic fatigue syndrome: An evidence-based approach to diagnosis and management by clinicians. Rev Environ Health 30: 223-249.

4. Cortes Rivera M, Mastronardi C, Silva-Aldana CT, Arcos-Burgos M, Lidbury BA (2019) Myalgic encephalomyelitis/chronic fatigue syndrome: A comprehensive review. Diagnostics (Basel) 9: E91.

5. Jason LA, Corradi K, Torres-Harding S, Taylor RR, King C (2005) Chronic fatigue syndrome: The need for subtypes. Neuropsychol Rev 15: 29-58.

6. Natelson BH, Lin JS, Lange G, Khan S, Stegner A, et al. (2019) The effect of comorbid medical and psychiatric diagnoses on chronic fatigue syndrome. Ann Med 51: 371-378.

7. Morris G, Maes M (2014) Mitochondrial dysfunctions in myalgic encephalomyelitis/chronic fatigue syndrome explained by activated immuno-inflammatory, oxidative and nitrosative stress pathways. Metab Brain Dis 29: 19-36.

8. Morris G, Anderson G, Maes M (2017) Hypothalamic-pituitary-adrenal hypofunction in myalgic encephalomyelitis (ME)/chronic fatigue syndrome (CFS) as a consequence of activated immune-inflammatory and oxidative and nitrosative pathways. Mol Neurobiol 54: 6806-6819.

9. Missailidis D, Annesley SJ, Fisher PR (2019) Pathological mechanisms underlying myalgic encephalomyelitis/chronic fatigue syndrome. Diagnostics (Basel) 9: E80

10. Naviaux RK, Naviaux JC, Li K, Bright AT, Alaynick WA, et al. (2016) Metabolic features of chronic fatigue syndrome. Proc Natl Acad Sci USA 113: E5472-E5480.

11. Fluge $\varnothing$, Mella $O$, Bruland $O$, Risa K, Dyrstad SE, et al. (2016) Metabolic profiling indicates impaired pyruvate dehydrogenase function in myalgic encephalopathy/chronic fatigue syndrome. JCl Insight 1: e89376.

12. Blomberg J, Gottfries CG, Elfaitouri A, Rizwan M, Rosén A (2018) Infection elicited autoimmunity and myalgic encephalomyelitis/chronic fatigue syndrome: An explanatory model. Front Immunol 9: 229.

13. Sotzny F, Blanco J, Capelli E, Castro-Marrero J, Steiner S, et al. (2018) Myalgic encephalomyelitis/chronic fatigue syndrome - Evidence for an autoimmune disease. Autoimmun Rev 17: 601-609.

14. Ryabkova VA, Churilov LP, Shoenfeld Y (2019) Neuroimmunology: What role for autoimmunity, neuroinflammation, and small fiber neuropathy in fibromyalgia, chronic fatigue syndrome, and adverse events after human papillomavirus vaccination? Int J Mol Sci 20: E5164.

15. Hornig M, Gottschalk CG, Eddy ML, Che X, Ukaigwe JE, et al. (2017) Immune network analysis of cerebrospinal fluid in myalgic encephalomyelitis/chronic fatigue syndrome with atypical and classical presentations. Transl Psychiatry 7: e1080.

16. Cliff JM, King EC, Lee JS, Sepúlveda N, Wolf AS, et al. (2019) Cellular immune function in myalgic encephalomyelitis/chronic fatigue syndrome (ME/CFS). Front Immunol 10: 796

17. de Vega WC, Herrera S, Vernon SD, McGowan PO (2017) Epigenetic modifications and glucocorticoid sensitivity in myalgic encephalomyelitis/chronic fatigue syndrome (ME/ CFS). BMC Med Genomics 10: 11.

18. Scheibenbogen C, Freitag H, Blanco J, Capelli E, LacerdaE, Authier J, et al. (2017) The European ME/CFS Biomarker Landscape project: An initiative of the European network EUROMENE. J TransI Med 15: 162.

19. Corbitt M, Eaton-Fitch N, Staines D, Cabanas H, Marshall-Gradisnik S (2019) A systematic review of cytokines in chronic fatigue syndrome/myalgic encephalomyelitis/systemic exertion intolerance disease (CFS/ME/SEID). BMC Neurol 19: 207.

20. Lidbury BA, Kita B, Lewis DP, Hayward S, Ludlow H, et al. (2017) Activin B is a novel biomarker for chronic fatigue syndrome/myalgic encephalomyelitis (ME/CFS) diagnosis: A cross sectional study. J Transl Med 15: 60.

21. Niblett SH, King KE, Dunstan RH, Clifton-Bligh $P$, Hoskin LA, et al. (2007) Hematologic and urinary excretion anomalies in patients with chronic fatigue syndrome. Exp Biol Med 232: 1041-1049.

22. Hornig M, Montoya JG, Klimas NG, Levine S, Felsenstein $D$, et al. (2015) Distinct plasma immune signatures in ME/ CFS are present early in the course of illness. Sci Adv 1: e1400121.

23. Montoya JG, Holmes TH, Anderson JN, Maecker HT, Rosenberg-Hasson Y, et al. (2017) Cytokine signature associated with disease severity in chronic fatigue syndrome patients. Proc Natl Acad Sci USA 114: E7150-E7158.

24. Brenu EW, van Driel ML, Staines DR, Ashton KJ, Ramos SB, et al. (2011) Immunological abnormalities as potential biomarkers in chronic fatigue syndrome/myalgic encephalomyelitis. J Transl Med 9: 81.

25. Fang $H$, Xie $Q$, Boneva $R$, Fostel J, Perkins R, Tong W (2006) Gene expression profile exploration of a large dataset on chronic fatigue syndrome. Pharmacogenomics 7 : 429-440.

26. Esfandyarpour R, Kashi A, Nemat-Gorgani M, Wilhelmy J, Davis RW (2019) A nanoelectronics-blood-based diagnostic biomarker for myalgic encephalomyelitis/chronic fatigue syndrome (ME/CFS). Proc Natl Acad Sci U S A 116: 1025010257.

27. Hyde B (1992) The clinical and scientific basis of myalgic encephalomyelitis - chronic fatigue syndrome. Nightingale Research Foundation Press, Ottawa, Canada.

28. Johnson H (1996) Osler's web: Inside the labyrinth of the chronic fatigue syndrome epidemic. iUniverse Inc., Lincoln, NE.

29. Martin WJ, Anderson D (1997) Stealth virus epidemic in the Mohave Valley. I. Initial report of viral isolation. Pathobiology 65: 51-56.

30. Martin WJ, Anderson D (1999) Stealth virus epidemic in the 
Mohave Valley: Severe vacuolating encephalopathy in a child presenting with a behavioral disorder. Exp Mol Pathol 66: $19-30$.

31. Glass RT (2000) Abnormal signs found in animals of myalgic encephalomyelitis/chronic fatigue syndrome patients. Journal of Chronic Fatigue Syndrome 6: 73-81.

32. Glass RT (2000) The human/animal interaction in myalgic encephalomyelitis/chronic fatigue syndrome. Journal of Chronic Fatigue Syndrome 6: 65-72.

33. Martin WJ, Glass RT (1995) Acute encephalopathy induced in cats with a stealth virus isolated from a patient with chronic fatigue syndrome. Pathobiology 63: 115-118.

34. Martin WJ (1992) Detection of viral related sequences in CFS patients using the polymerase chain reaction. In: Byron $\mathrm{M}$ Hyde, The clinical and scientific basis of myalgic encephalomyelitis chronic fatigue syndrome. Nightingale Research Foundation Press, Ottawa, Canada, 278-283.

35. Martin WJ (1992) Viral infection in CFS patients. In: Byron $M$ Hyde, The clinical and scientific basis of myalgic encephalomyelitis chronic fatigue syndrome. Nightingale Research Foundation Press, Ottawa, Canada, 325-327.

36. Martin WJ, Zeng LC, Ahmed K, Roy M (1994) Cytomegalovirus-related sequences in an atypical cytopathic virus repeatedly isolated from a patient with the chronic fatigue syndrome. Am J Path 145: 440-451.

37. Martin WJ (1994) Stealth viruses as neuropathogens. CAP Today 8: $67-70$

38. Martin WJ (1996) Severe stealth virus encephalopathy following chronic fatigue syndrome-like illness: Clinical and histopathological features. Pathobiology 64: 1-8.

39. Martin WJ (1996) Stealth viral encephalopathy: Report of a fatal case complicated by cerebral vasculitis. Pathobiology 64: 59-63.

40. Martin WJ (2104) Stealth Adapted Viruses; Alternative Cellular Energy (ACE) \& KELEA Activated Water. Author-
House, Bloomingdale, MN.

41. Martin WJ, Ahmed KN, Zeng LC, Olsen J-C, Seward JG, et al. (1995) African green monkey origin of the atypical cytopathic 'stealth virus' isolated from a patient with chronic fatigue syndrome. Clin DiagVirol 4: 93-103.

42. Martin WJ (1996) Simian cytomegalovirus-related stealth virus isolated from the cerebrospinal fluid of a patient with bipolar psychosis and acute encephalopathy. Pathobiology 64: 64-66.

43. Martin WJ (1996) Genetic instability and fragmentation of a stealth viral genome. Pathobiology 64: 9-17.

44. Martin WJ (1998) Cellular sequences in stealth viruses. Pathobiology 66: 53-58.

45. Martin WJ (1999) Bacteria related sequences in a simian cytomegalovirus-derived stealth virus culture. Exp Mol Path 66: 8-14.

46. Martin WJ (2019) Renegade cellular and bacterial genetic sequences in monkey-derived stealth adapted viruses. J Human Virol \& Retrovirology 7: 26-40.

47. Endresen G (2003) Mycoplasma blood infection in chronic fatigue and fibromyalgia syndromes. Rheumatol Int 23: 211-215.

48. Alter HJ, Mikovits JA, Switzer WM, Ruscetti FW, Lo SC, et al. (2012) A multicenter blinded analysis indicates no association between chronic fatigue syndrome/myalgic encephalomyelitis and either xenotropic murine leukemia virus-related virus or polytropic murine leukemia virus. MBio 3: e00266-e00312.

49. Martin WJ (2015) Is the brain an activator of the alternative cellular energy (ACE) pathway? Int J Complement Alt Med 1: 6-9.

50. Martin WJ (2020) Enhancing the alternative cellular energy (ACE) pathway with KELEA activated water as therapy for infectious diseases. Infectious Diseases - Drug Targets (in press). 